\title{
Capital intelectual en la gestión pública: caso del método Intellectus
}

\begin{abstract}
RESUMEN
Este artículo tiene el objetivo de comprobar la cohesión de los temas capital intelectual y gestión pública por medio de las percepciones de gerentes públicos sobre terminologías específicas de capital intelectual. La investigación ha usado como base el modelo llamado Intellectus, en la cual se ha desarrollado una investigación con gerentes públicos participantes de un programa de maestría en gestión de políticas públicas. Los resultados demuestran la viabilidad de implantación y el modelo propuesto puede ser la base para futuras generalizaciones. Los encuestados tuvieron pocas dudas y lograron fijar sus percepciones en escala cualitativa. La opción por un análisis desde la media aritmética ocurrió por la facilidad de comprensión de quien fue aplicar el modelo y utilizar las informaciones resultantes para promover nuevas investigaciones y aplicaciones.
\end{abstract}

Palabras clave: Gestión Pública. Intellectus. Capital Intelectual.

Marco Antonio Harms Dias mdias@univali.br

Professor e Pesquisador - Universidade do Vale do Itajaí. Doutorando em Engenharia e Gestão do Conhecimento - UFSC Alexandre Avila Lerípio leripio@terra.com.br Universidade do Vale do Itajaí Gregório Rados Varvakis grego@deps.ufsc.br

Universidade Federal de Santa Catarina Caroline Rodrigues Vaz karolrvaz@gmail.com Pesquisadora do NGS/UFSC. Doutoranda em Engenharia de Produção - UFSC 


\section{INTRODUCCIÓN}

Así como en todas las organizaciones, la gestión pública, con el advenimiento de la crisis del Estado-nación y la emergencia de la sociedad del conocimiento (DRUCKER, 2002) frente a la sucesión del modelo industrial (BUENO et al, 2004), confrontase con la necesidad de respuestas a cuestionamientos sobre cómo gestionar programas públicos intensivos en conocimiento estructurados sobre recursos intangibles y en contextos adversos a las reglas del mercado.

Por lo que se sabe, en el sector público hay el concepto de valor público (MOORE, 2007) y el objetivo de las acciones de gestión pública residen en su entrega a los ciudadanos de forma superior, con semejanzas a las iniciativas de gestión reguladas por prácticas de mercado (BOYNE, 2002; PARK, 2007; WIIG, 2002).

Por otro lado, se sabe que las implantaciones de técnicas de gerencialismo en el sector público fueron iniciadas en las últimas décadas y se encontraron con dificultades sui generis del ambiente público, principalmente frente a las diferencias entre las formas de gestión, relaciones con stakeholders y sus finalidades (BUENO et al, 2004; DENHARDT, 2008; MATIAS-PEREIRA, 2010; RAMIREZ, 2010).

En ese sentido, también emerge en la literatura y prácticas empresariales, la gestión de activos de conocimiento y de intangibles que, para autores como Bueno et al (2011), Bontis (1999) y Edvinson y Mallone (1998) entre otros, pueden ser caracterizados como capital intelectual. Ese conjunto de valores, generadores de riqueza, poseen diálogo conceptual con valor público (MOORE, 2007) y contextual con la gestión pública, según Matias-Pereira (2010) y Ramirez (2010).

Frente a las limitaciones y el concepto de una administración pública emergente, según Denhardt (2008) y Bueno et al (2004), se propone en ese trabajo comprobar la cohesión de los temas capital intelectual y gestión pública por medio de las percepciones de gerentes públicos sobre terminologías específicas de capital intelectual.

Desde las respuestas se podrá evaluar caminos y posibilidades de implantación y desarrollo de nuevas investigaciones involucrando los temas, bien como la concepción de herramientas de gestión de capital intelectual específicas al contexto público.

Ese artículo está estructurado en cinco secciones, la primera está compuesta de esta introducción, la segunda sección trae un levantamiento teórico que fundamenta capital intelectual y gestión pública, la tercera sección presenta el guión de las encuestas y los procedimientos metodológicos, la cuarta sección enseña un análisis de los resultados obtenidos con la colecta de los datos y termina con la quinta sección y la presentación de las consideraciones finales.

\section{REVISIÓN TEÓRICA}

Este capítulo se presenta en tres partes, una primera contextualización del objeto de estudio, el capital intelectual, desde su concepción, la evolución conceptual, su relación con la gestión del conocimiento y la relevancia del tema en estos días. Después, se presenta una revisión del modelo de gestión y el capital intelectual, intellectus, demostrando sus propiedades y características, un relato de sus aplicaciones y por lo tanto su elección en este estudio. Cierra este capítulo en el "locus" de la investigación, en este caso, el campo teórico de la gestión pública, la forma sintética se detalla su participación en los debates actuales de la sociedad del conocimiento, así como los esfuerzos para modernizar a las técnicas de gestión.

\subsection{Capital Intelectual}

Principalmente desde la década de 1990, el tema capital intelectual surge en la literatura y prácticas empresariales, debido a la comprensión de que "en la presente economía, más y más negocios están conectados a valores no enlazados a los recursos tangibles, sino a sus recursos intangibles" (BONTIS, 1999, p. 435).

El capital intelectual "constituye la materia intelectual - conocimiento, información, propiedad intelectual, experiencia que puede ser utilizada para generar riqueza. Es la capacidad mental colectiva". Luego, se admite que el Capital Intelectual, como el conjunto de valores ocultos que añaden valor a las organizaciones, permitiendo su continuidad (STEWART, 1998).

Consideran Edvinsson y Sullivan (1996) el capital intelectual como el conocimiento que puede ser convertido en valor, abarcando invenciones, ideas, conocimientos generales, proyectos, programas 
computacionales, procesos y publicaciones.

El capital intelectual es un capital no financiero que representa la brecha oculta entre el valor de mercado y el valor contable. Siendo, segundo Edvinson y Sullivan (1996) y Edvinsson y Malone (1998), por ejemplo, a suma del Capital Humano y del Capital Estructural. En esa visión, el capital humano corresponde a toda la capacidad, conocimiento, habilidad y experiencia individuales de los empleados de una organización para realizar las tareas, mientras que el capital estructural se forma por la infraestructura que apoya el capital humano, en otras palabras, todo lo que permanece en la empresa cuando los empleados van para casa.

Diversos autores presentan formas de analizarse y evaluar el capital intelectual desde estudios iniciales y, hoy, con el tema de algún modo consolidado (EDVINSSON, 2013), hay consenso de la estructuración de ese recurso en las organizaciones desde tres dimensiones: humana, estructural y social (BUENO et al, 2011).

En síntesis segundo Sveiby (1998) y Bueno et al (2011), se puede describir la dimensión humana como la representación del saber hacer, la dimensión estructural como los conocimientos que quedan en la organización en la ausencia de los empleados y la dimensión social como las relaciones de la organización con sus diversos stakeholders.

Con foco en la estrategia volteada hacia el recurso (DUMAY, 2013), el capital intelectual demuestra una forma de gestionar el conocimiento como el recurso valioso de una organización, sin embargo al contrario de activos físicos, activos de conocimiento, también conocidos como capital intelectual, son mucho más difíciles de cuantificar.

Entre diversos modelos de medición de gestión de capital intelectual, se elige en este estudio el Modelo Intellectus por ser caracterizado como sistémico, abierto, flexible, adaptativo y dinámico (BUENO et al, 2011).

\subsection{Modelo Intellectus}

Modelo Intellectus desarrollado por la Universidad Autónoma de Madrid surgió después de la mejora del modelo Intelect en 2003 y de experiencias en diversos contextos y desarrollo de investigaciones aplicadas, con la incorporación de mejoras en la capacidad operatoria en la práctica.

El modelo Intellectus tiene por objetivo la medición y la gestión de valores intangibles y del conocimiento que componen el concepto de capital intelectual. El modelo cuenta con cinco puntos relativos a cada una de las partes principales de la propuesta, siendo: estructura, principios (características), lógica interna, desarrollo del modelo (definiciones), cuadro de indicadores.

La estructura del modelo Intellectus se divide en cuatro conceptos básicos, siendo ellos:

a. Componentes o Capitales: conjunto de activos intangibles en función de su naturaleza;

b. Elementos: grupos homogéneos de activos intangibles de cada uno de los componentes del capital intelectual;

c. Variables: activos intangibles miembros de un elemento del capital intelectual;

d. Indicadores: instrumentos de valorización de los activos intangibles de las organizaciones.

En relación a sus características el modelo se presenta como:

a. Sistémico: ofrece una estructura interrelacionada y completa de los cincos aspectos para que venga a representar los componentes y subsistemas principales que configuran los elementos y variables explicativas de los activos intangibles e intelectuales;

b. Abierto: presenta una estructura relacionada con los agentes, el sujeto de conocimiento que integran el entorno de la organización y que se explica por un conjunto de relaciones con lo que se pueden mantener aquellas personas que la componen;

c. Dinámico: ofrece un conjunto de elementos, variables, indicadores y relaciones que deben permitir la observación de su evolución temporal, con el objetivo de ir logrando una mejora en la gestión de las actividades intangibles y un mayor valor de los componentes del capital intelectual de una organización;

d. Flexible: los elementos y variables propuestas pueden ser ordenados y aplicados de forma diferencial según las necesidades de la organización;

e. Adaptable: requiere de una regla generalmente adaptada para su aplicación a determinadas tipologías organizacionales, siendo cuales sean las características propias de la actividad económica;

f. Innovador: presenta una perspectiva moderna, completa y original, ubicando en un plano de interés económico que quiere propiciar los procesos de creación, medición y gestión de intangibles.

La lógica interna es la parte interna del modelo, que enseña la aceptación del carácter dinámico del modelo Intellectus, de su capacidad evolutiva que facilita la comprensión de su funcionamiento, 
no como una propuesta dogmática, pero sí, como manera de garantizar alguna consistencia entre los elementos explicativos de los diferentes activos intelectuales y las relaciones al funcionamiento que las conectan.

Para el desarrollo del modelo, son realizadas las definiciones de los elementos: capital humano (valores y actitudes), capital estructural (capital organizacional - cultura, estructura, aprendizaje, proceso y; tecnológico - esfuerzo, asignación, propiedad intelectual e industrial), capital relacional (capital de negocio - relación con clientes, proveedores, accionistas, aliados, competidores, administradores y; capital social - relaciones con la administración pública, imagen corporativa, defesa con medio ambiente, relaciones sociales). Y por fin, las organizaciones dibujan sus indicadores que pueden ser ajustados a sus necesidades en el modelo de capital intelectual, con una serie de principios y características con la meta de asegurar alguna homogeneidad en su aplicación práctica.

Correctamente consolidado después de diversos estudios y aplicaciones el Modelo Intellectus se estructura de manera jerárquica, de cuatro niveles desde tres capitales, sin embargo se presenta con seis capitales: más allá del Humano, el capital organizativo y tecnológico fueron desmembrados desde el capital estructural y los capitales de negocio y relacional desde el capital social. El sexto capital, el de emprendimiento e innovación, fue concebido para enseñar como actúan los aceleradores de los procesos de generación de valor. Los cuatro niveles jerárquicos del modelo Intellectus son: los capitales, los elementos, variables e indicadores, como establecido en la figura 1.

Figura 1 - Modelo Intellectus de Capital Intelectual

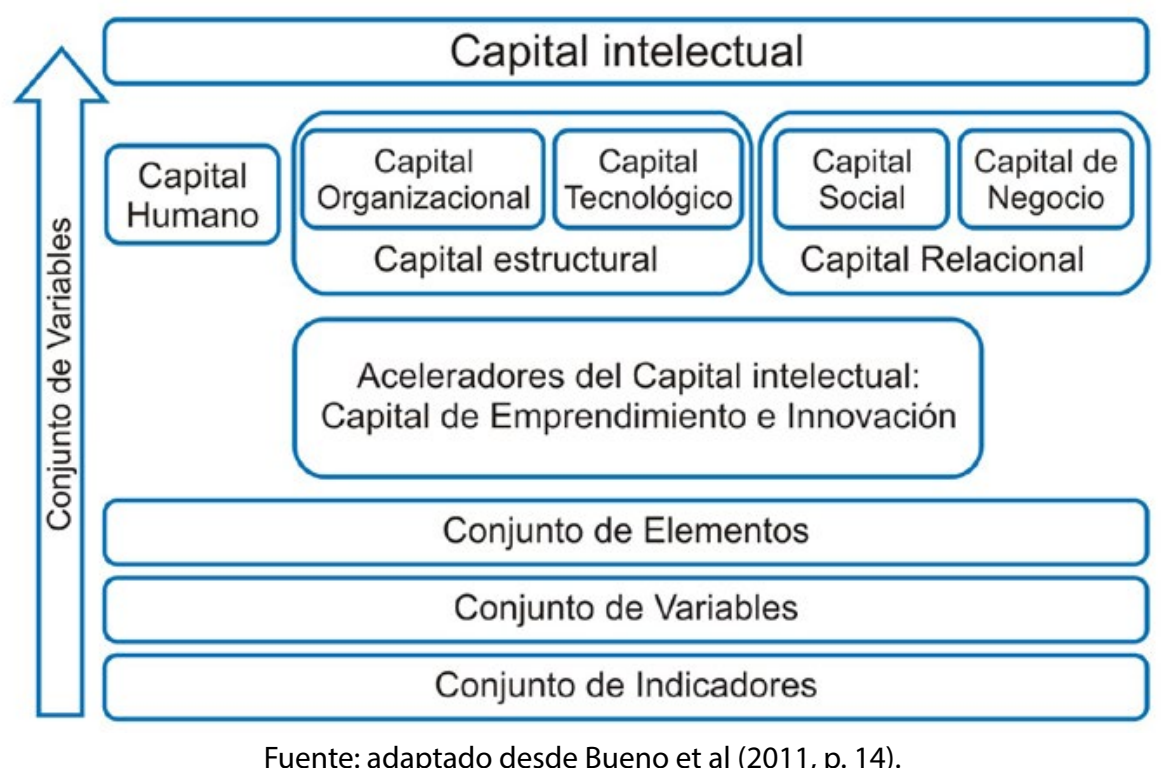

El modelo Intellectus fue aplicado en gestión pública como las experiencias de Bueno et al (2004), y los supuestos de esos autores, desde la aplicación en el Ministerio de fiscalización de impuestos español, son presentados en Bueno, Salmador y Merino (2006). Como se caracteriza por un modelo dinámico, con nuevas experiencias, principalmente en empresas mercantiles, fueron agregadas, culminando en un modelo estructurado según detallado en Bueno et al (2011). Una síntesis de las diferencias en estos dos períodos, se encuentra en la figura 2. 
Figura 2 - Modelo Intellectus - diferencias entre Bueno, Salmador y Merino (2006) y Bueno et al (2011)

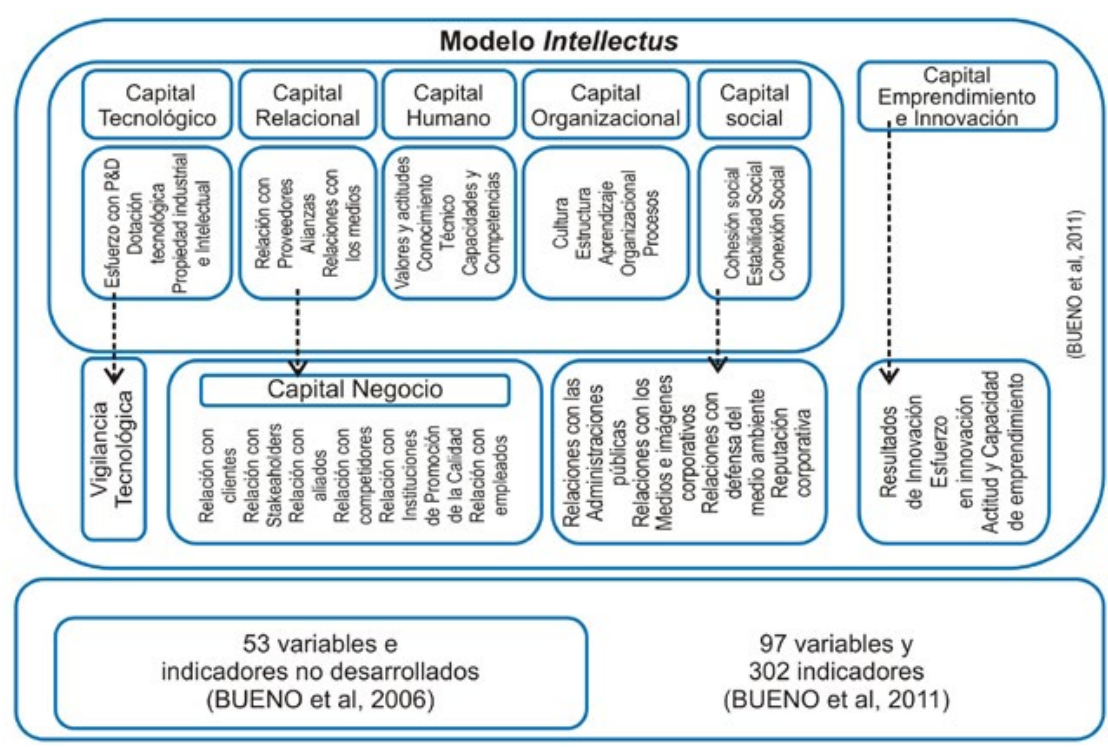

Fuente: elaborado por los autores (2014)

Se nota que hay necesidad de complementación y actualización de la propuesta del modelo Intellectus para la gestión pública presentado en Bueno, Salmador y Merino (2006), con la ubicación y contextualización de los elementos y variables a la luz de Bueno et al (2011) y la percepción de los gerentes públicos.

\subsection{Gestión Pública}

La gestión pública pasa por un momento de transición entre el modelo burocrático para un modelo debidamente adaptado a un mundo interdependiente y en constante cambio (MATIASPEREIRA, 2010). Los intentos originados principalmente en la década de los años 1980 de inserción de prácticas venidas del mundo empresarial para el alcance de la gestión pública enseñó ineficacia debido a diversos factores (DENHARDT, 2008; BUENO et al, 2004), principalmente en simple conversión y adaptación de los modelos y en las búsquedas de similitudes entre los conceptos de ciudadanos y clientes (BUENO et al, 2004; BOYNE, 2002).

En este sentido, el movimiento llamado "new public management" fue establecido en busca de una modernización de la administración pública y, entre las críticas y limitaciones, autores apuntan la deficiencia en los paralelos entre los conceptos de cliente y ciudadanos. Desde ese movimiento nuevos conceptos surgieron "especialmente los relacionados con eficiencia, eficacia y contribución positiva en la elaboración de una visión más gerencial de la gestión gubernamental" (RAMIREZ, 2010, p. 250).

Esos conceptos traen aspectos de gestión conectados a la competitividad y, a pesar de "no usualmente buscar ventajas competitivas" (SERVIN, 2005, p. 9), la gestión pública busca apoyo y aplicación de conceptos como recursos valiosos, como por ejemplo, la importancia del servicio público (DENHARDT, 2008) entregar valor público (MOORE, 2007).

Por ejemplo, en las obras de la $\operatorname{OECD}(2001,2008)$ existe una clara tendencia en la incorporación de tecnologías de la información y comunicación en la gestión pública, y autores como Abma y Noordegraaf (2003) defensores de que hay necesidad de especificidad a la gestión pública al afirmaren que no hay como hacer una simple correlación en la aplicación de medidas de rendimiento entre los modelos de gestión privados y públicos.

En ese sentido se concluye que para proponer un instrumento de gestión para el capital intelectual, hay necesidad de buscarse adaptaciones a las especificidades de una gestión compleja como es la que envuelve las cuestiones públicas.

\section{METODOLOGÍA}

Este capítulo se divide en tres presentes sub-capítulos, el primero con la investigación, seguida de los detalles de los métodos analíticos y, por último, una descripción de los sujetos de investigación. 


\subsection{Caracterización de la investigación}

Caracterizado como un estudio de caso, buscando a la generalización de las reflexiones para nuevos contextos desde una realidad subjetiva se pretende con esa investigación cualitativa (DENZIN; LINCOLN, 2006) caracterizada como básica o genérica (MERRIAN, 1998), descubrir o desvelar cuestiones inherentes al capital intelectual en gestión de programas públicos desde la adecuación del modelo Intellectus al contexto vivido por gerentes públicos.

\subsection{Métodos de Recopilación y Análisis de Datos}

El levantamiento de los datos ocurrió por medio de encuesta por cuestionario elaborado según los elementos y variables del modelo Intellectus (BUENO et al, 2011), compuesto por cuestiones con escalas cualitativas, predominantemente por escalas de importancia - del "extremadamente importante" al "nada importante" y likert - del "desacuerdo totalmente" al "acuerdo totalmente" (FAVERO et al, 2009),

De esa manera se buscó cuestionar la pertinencia de cada una de las variables, desde el presupuesto que no teniendo pertinencia o una duda de la aplicación al contexto público enseñará falta de cohesión entre Capital Intelectual y Gestión Pública.

Las 97 variables presentadas en Bueno et al (2011) fueron evaluadas a la luz de los principios de la gestión pública, según Denhardt (2008), para que se borren las variables que no tenían relación conceptual y de aplicación con eses principios. Resultó segundo la percepción de los autores, en 71 cuestiones adherentes a la complejidad de la gestión pública.

En el instrumento de coleta, más allá de las respuestas en las escalas, fue solicitado a los sujetos sus impresiones y dudas sobre cada una de las 71 variables, por medio de espacio al lado de cada respuesta. En la aplicación fue entregue con una carta de presentación, describiendo los objetivos de la investigación, explicando el criterio de su selección para la encuesta y orientaciones. Las variables fueron divididas en grupos, según el elemento que las componga, con una breve explicación de lo que representaba ese conjunto.

\subsection{Sujetos de la Investigación}

La investigación fue realizada con 10 gerentes públicos matriculados en un curso de maestría en gestión de políticas públicas, ocurriendo la intervención en el comienzo de una actividad lectiva regular. Todos asignaron valores a todas las 71 variables presentadas y sólo cuatro sujetos demostraron dudas en os cuestionamientos, con la explicitación en campo de comentarios al lado del cuestionamiento.

Los comentarios fueron de duda para la variable "homogeneidad cultural", que componen el elemento "cultura" dentro del Capital Organizativo, uno de los comentarios afirmaba la dificultad de ocurrir y de medirse esa variable en una gestión pública.

\section{ANÁLISIS Y DISCUSIÓN DE LOS RESULTADOS}

Siendo el objetivo de esa investigación comprobar con cohesión conceptual entre capital intelectual y gestión pública, por haber tenido respuestas a todos los cuestionamientos y las dudas perteneceren sólo en la aplicación y no en la incoherencia, se nota en principio tratamiento a los presupuestos de la investigación.

Se buscó para una mejor comprensión para tratar a los preceptos del Modelo Intellectus de demostrar la forma orgánica y sistémica de sus datos, la integración de los datos obtenidos en el sentido de buscarse una lógica de percepción de los gerentes sobre ese conjunto de intangibles en el contexto público. Por lo tanto se redujo la realidad percibida por medio de medias estadísticas, según se presenta.

Las medias de las respuestas, posibles en una escala entendida entre 1 e 5 , quedaron en 4,2. Demostrando alto grado de pertinencia. Después del cálculo de las medias de todas las 71 variables, se compuso las medias de cada elemento y, en secuencia, el valor medio del capital por la media de los valores de los elementos. Teniendo las medias de los valores, se llegó a los pesos de las variables, de los elementos y de los capitales. Segundo sintetizado en la figura 3. 
Figura 3 - Peso de los capitales

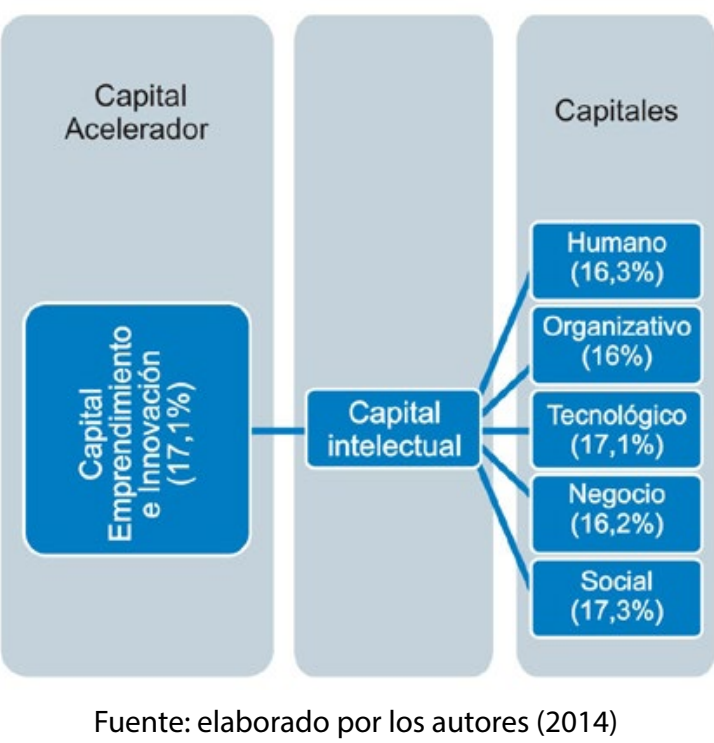

Recordando que la estructura del Intellectus de forma jerárquica contiene conjuntos de variables que forman un elemento y el conjunto de elementos forman los componentes o capitales, se levantó desde las medias de cada una de las variables, el peso de distribución de las pertinencias de cada capital, respetando la debida proporcionalidad.

La distribución de las medias y pesos de los elementos, para cada capital, está representada en el cuadro 1.

Cuadro 1- Distribución de las medias y pesos de los elementos, por capital

\begin{tabular}{|c|c|c|c|}
\hline Capital & Elemento & $\begin{array}{c}\text { Media de } \\
\text { los valores }\end{array}$ & $\begin{array}{c}\text { Pesos } \\
\text { (en \%) }\end{array}$ \\
\hline Humano & Valores y actitudes & 4,2 & 34 \\
\hline Humano & Habilidades & 4,1 & 33,2 \\
\hline Humano & Capacidades & 4,1 & 32,8 \\
\hline Organizativo & Cultura & 4,0 & 48,6 \\
\hline Organizativo & Aprendizaje Organizacional & 4,2 & 51,4 \\
\hline Tecnológico & Esfuerzo de P\&D & 4,3 & 49,4 \\
\hline Tecnológico & Tecnología & 4,4 & 50,6 \\
\hline Negocio & Relaciones con stakeholders - usuarios & 4,0 & 16 \\
\hline Negocio & Relaciones con proveedores & 3,7 & 15 \\
\hline Negocio & Relaciones con stakeholders - patrocinadores & 4,4 & 17,5 \\
\hline Negocio & Relaciones con aliados & 4,2 & 16,9 \\
\hline Negocio & Relaciones con instituciones - calidad & 4,5 & 17,9 \\
\hline Negocio & Relaciones con empleados & 4,2 & 16,5 \\
\hline Social & Relaciones con diversas esferas & 4,3 & 49,2 \\
\hline Social & Reputación corporativa & 4,5 & 50,8 \\
\hline $\begin{array}{c}\text { Emprendimiento e } \\
\text { Innovación }\end{array}$ & Resultados de la innovación & 4,3 & 32,9 \\
\hline $\begin{array}{c}\text { Emprendimiento e } \\
\text { Innovación }\end{array}$ & Esfuerzo en innovación & 4,4 & 33,4 \\
\hline $\begin{array}{c}\text { Emprendimiento e } \\
\text { Innovación }\end{array}$ & Actitudes y Capacidad de emprendimiento & 4,4 & 33,7 \\
\hline
\end{tabular}

Fuente: elaborado por los autores (2014) 
Desde esas medias de valores y pesos puede darse cuenta como lo que cada una de las variables, elementos y capitales se distribuyen de acuerdo con la pertinencia, permitiendo nuevos estudios para, por ejemplo, evaluar los temas más importantes, o la integración de conceptos que el Intellectus separa o, por otro lado, separa aspectos a veces integrados.

\section{CONSIDERACIONES FINALES}

Con el estudio realizado se nota que es posible adecuar y contextualizar el modelo Intellectus a la gestión pública segundo las percepciones de los gerentes. En esta investigación se levantó informaciones con un selecto grupo de gerentes públicos, debidamente insertados en ambiente académico, sin embargo hay evidencias por los resultados que hay adherencia del modelo estudiado con la práctica de la gestión pública.

Los encuestados tuvieron pocas dudas y consiguieron fijar sus percepciones en escala cualitativa. La opción por un análisis desde la media aritmética se dió por la facilidad de comprensión de quien fue aplicar el modelo y utilizar las informaciones resultantes para promover nuevas investigaciones y aplicaciones.

Se nota que mientras la reputación corporativa, en el Capital Social, y las relaciones con instituciones de calidad, en el Capital de Negocio presentan las mayores medias de los valores con 4,5 , el elemento "relaciones con proveedores", en el Capital negocio, presentó la menor media, con 3,7. La cultura, en el Capital Organizativo, y las relaciones con stakeholders - usuarios obtuvieron medias de 4,0 .

También es relevante la media superior del Capital Social en la composición del Capital Intelectual por la percepción de los sujetos de la investigación, en un programa público.

Desde ese estudio que presenta las medias y ponderaciones de las variables, elementos y capitales para la composición del capital intelectual en la gestión pública, se cree en la posibilidad de aplicación de un modelo de análisis del capital intelectual con el desarrollo de nuevos pasos, nombradamente la proposición de indicadores que alimentarán la base de los datos para, con los pesos, presentar números que representan los capitales componentes del capital intelectual del programa público estudiado.

\section{INTELLECTUAL CAPITAL IN PUBLIC ADMINISTRATION: THE CASE METHOD Intellectus}

\section{ABSTRACT}

This article aims to verify the cohesion of intellectual capital and governance issues through the perceptions of public managers about specific terminologies related to intellectual capital. This research has used as a base a model called Intellectus, in which a research with public managers was carried out by participants of a Master's Degree program in management of public policies. The results demonstrate the feasibility of its implementation and the possibility for the proposed model to become the basis for further generalizations. Respondents had few doubts and managed to place their perceptions in a qualitative scale. The option for an analysis based on arithmetic average occurred due to the ease of interpretation by those who applied the model and used the resulting information to promote further research and applications.

Keywords: Public management. Intellectus. Intellectual Capital. 


\section{REFERENCIAS}

ABMA, T. A.; NOORDEGRAAF, M. Public Managers amidst Ambiguity: towards a typology of evaluative practices in public management. Evaluation, London, v. 9, n. 3, p. 285-306, 2003.

BONTIS, N. Managing Organizational Knowledge by Diagnosing Intellectual Capital: framing and advancing the state of the field. International Journal Techonology Management, v. 18, n. 5, p. 433-463, 1999.

BOYNE, G.A. Public and Private Management: what's the difference? Journal of Management Studies, v. 39, n. 1, jan. 2002.

BUENO, E. et al (Org.) . La Administración Pública como Agente de Conocimiento en la Sociedade de la Información. Madrid: Universidad Autónoma de Madrid, 2004.

BUENO, E.; SALMADOR, M.P; MERINO, C. Towards a model of intellectual capital in public administrations. International Journal Learning and Intellectual Capital, v. 3, n. 3, p. 214-232, 2006.

BUENO, E. et al. Propuesta de Nuevo Modelo Intellectus de Medición, Gestión e Información del Capital Intelectual. Madrid: Universidad Autónoma de Madrid, 2011.

DENHARDT, Robert B. Theories of Public Organization. 5th ed. Belmont, CA:Thomson, 2008.

DENZIN, N.K.; LINCOLN, Y.S. O Planejamento da Pesquisa Qualitativa: teorias e abordagens. Porto Alegre: Artmed, 2006.

DRUCKER, P. Sociedade pós-capitalista. São Paulo: Pioneira, 2002.

DUMAY, J. The Third Stage of IC: towards a new IC future and beyond. Journal of Intellectual Capital, v. 14, n. 1, p. 5-9, 2013.

EDVINSSON, L. IC 21: reflections from 21 years of IC practice and theory. Journal of Intellectual Capital, v. 14, n. 1, p. 163-172, 2013.

EDVINSSON, L.; MALONE, M. S. Capital Intelectual. São Paulo: Makron Books, 1998.

EDVINSSON, L.; SULLIVAN, P. H. Developing a model for managing intellectual capital. European Management Journal, v. 14, n. 4, p. 356-364, 1996.

FAVERO, L.P. et al. Análise de dados: modelagem multivariada para tomada de decisões. Rio Janeiro: Elsevier, 2009.

MATIAS-PEREIRA, J. Governança no setor público. São Paulo: Atlas, 2010.

MERRIAM, S. B. Qualitative research and case study applications in education. San Francisco: Jossey-Bass, 1998.

MOORE, M.H. Criando Valor Público Por Meio de Parcerias Público-Privadas. Revista do Serviço Público, v. 58, n. 2, p. 151-179, abr./jun. 2007.

ORGANISATION FOR ECONOMIC CO-OPERATION AND DEVELOPMENT (OECD). Governance in the 21 st Century. Paris: OECD Publishing, 2001.

ORGANIZACIÓN PARA LA COOPERACIÓN Y EL DESARROLLO ECONÓMICO (OECD). Evaluación de la Participación Pública em la Elaboración de Políticas Públicas. Madrid: Instituto Nacional de Administración Pública, 2008.

PARK, S. C. The Comparison of Knowledge Management Practices Between Public and Private Organizacionts: an exploratory study. Thesis (Doctor of Philosophy) - School of Public Affairs, Pennsylvania State University, 2007.

RAMIREZ, Y. Intellectual Capital Models in Spanish Public Sector. Journal of Intellectual Capital, v. 11, n. 2, p. 248-264, 2010. 
SERVIN, G. ABC of Knowledge Management. [2005]. Disponível em: <http://www.fao.org/ fileadmin/user_upload/knowledge/docs/ABC_of_KM.pdf>. Acesso em: 10 dez. 2014.

STEWART, T. A. Capital Intelectual: A nova vantagem competitiva das organizações. Rio de Janeiro: Campus, 1998.

SVEIBY, K. E. A Nova Riqueza das Organizações. Rio de Janeiro: Campus, 1998.

WIIG, K.M. Knowledge Management in Public Administration. Journal of Knowledge Management, v. 6, n. 2, p. 224-239, 2002. 